\title{
STING protects against cardiac dysfunction and remodelling by blocking autophagy
}

\author{
Rui Xiong ${ }^{1 \dagger}$, Ning $\mathrm{Li}^{1 \dagger}$, Lei Chen ${ }^{2 \dagger}$, Wei Wang ${ }^{1}$, Bo Wang ${ }^{1}$, Wenyang Jiang ${ }^{1 *}$ and Qing Geng ${ }^{1 *}$ (])
}

\begin{abstract}
Background: Heart failure, which is characterized by cardiac remodelling, is one of the most common chronic diseases in the aged. Stimulator of interferon genes (STING) acts as an indispensable molecule modulating immune response and inflammation in many diseases. However, the effects of STING on cardiomyopathy, especially cardiac remodelling are still largely unknown. This study was designed to investigate whether STING could affect cardiac remodelling and to explore the potential mechanisms.
\end{abstract}

Methods: In vivo, aortic binding (AB) surgery was performed to construct the mice model of cardiac remodelling. A DNA microinjection system was used to trigger STING overexpression in mice. The STING mRNA and protein expression levels in mice heart were measured, and the cardiac hypertrophy, fibrosis, inflammation and cardiac function were also evaluated. In vitro, cardiomyocytes stimulated by Ang II and cardiac fibroblasts stimulated by TGF- $\beta$ to performed to further study effects of STING on cardiac hypertrophy and fibroblast. In terms of mechanisms, the level of autophagy was detected in mice challenged with AB. Rapamycin, a canonical autophagy inducer, intraperitoneal injected into mice to study possible potential pathway.

Results: In vivo, the STING mRNA and protein expression levels in mice heart challenged with AB for 6 weeks were significantly increased. STING overexpression significantly mitigated cardiac hypertrophy, fibrosis and inflammation, apart from improving cardiac function. In vitro, experiments further disclosed that STING overexpression in cardiomyocytes induced by Ang II significantly inhibited the level of cardiomyocyte cross-section area and the ANP mRNA. Meanwhile, TGF- $\beta$-induced the increase of a-SMA content and collagen synthesis in cardiac fibroblasts could be also blocked by STING overexpression. In terms of mechanisms, mice challenged with AB showed higher level of autophagy compared with the normal mice. However, STING overexpression could reverse the activation of autophagy triggered by AB. Rapamycin, a canonical autophagy inducer, offset the cardioprotective effects of STING in mice challenged with AB. Finally, further experiments unveiled that STING may inhibit autophagy by phosphorylating ULK1 on serine 757.

Conclusions: STING may prevent cardiac remodelling induced by pressure overload by inhibiting autophagy, which could be a promising therapeutic target in heart failure.

Keywords: STING, Cardiac remodelling, Inflammation, Autophagy, ULK1

*Correspondence: whujwy0228@163.com; gengqingwhu@whu.edu.cn ${ }^{\dagger}$ Rui Xiong, Ning Li and Lei Chen contributed equally to the work.

${ }^{1}$ Department of Thoracic Surgery, Renmin Hospital of Wuhan University, Wuhan, China

Full list of author information is available at the end of the article

\section{Background}

Cardiac remodelling is defined as the abnormal alterations in cardiac structure, shape, and function stimulated by pathophysiologic condition, which is implicated with a great many cardiac disorders, involving myocardial ischemia, myocardial infarction, diabetic cardiomyopathy, hypertrophic cardiomyopathy, original author(s) and the source, provide a link to the Creative Commons licence, and indicate if changes were made. The images or other third party material in this article are included in the article's Creative Commons licence, unless indicated otherwise in a credit line to the material. If material is not included in the article's Creative Commons licence and your intended use is not permitted by statutory regulation or exceeds the permitted use, you will need to obtain permission directly from the copyright holder. To view a copy of this licence, visit http://creativecommons.org/licenses/by/4.0/. The Creative Commons Public Domain Dedication waiver (http://creativeco mmons.org/publicdomain/zero/1.0/) applies to the data made available in this article, unless otherwise stated in a credit line to the data. 
doxorubicin-induced cardiac fibrosis as well as heart failure [1-3]. In particular, cardiac remodelling causes the increased morbidity and mortality among the elderly each year, which leads to sever economy burden globally [4]. Mountainous evidence has proved that cardiomyocyte hypertrophy and abnormal activation of cardiac fibroblast are the key mechanisms contributing to cardiac remodeling [5]. Thus, genes and proteins which could selectively slow down the progression of cardiac fibrosis and hypertrophy would be of great therapeutic interest.

Autophagy performs housekeeping functions for some degradation and recycling of cytoplasmic proteins and organelles, which plays a crucial role in adverse cardiac remodelling to regulate cellular homeostasis and cardiac function in the heart [6]. Cardiomyocyte autophagy and cardiac fibroblast autophagy are the primary sources of cardiac remodelling, but the beneficial or aggravating role of the autophagy in cardiac remodelling remains controversial for the reason that both "excessive" and "insufficient" autophagy in heart are detrimental [7]. Further study needs to be carried out to illustrate the precise regulatory mechanisms of autophagy in cardiac remodelling.

Stimulator of interferon gene (STING) is an important regulator of DNA sensing pathways. It is physiologically embedded in the endoplasmic reticulum and induces the production of type I interferon by activating nuclear factor (NF) $-\mathrm{kB}$ and interferons (IFN) regulator 3 (IRF3) pathways [8]. Previous study has demonstrated that in live Gram-positive bacteria, STING could sense cell viability to modulate pathogen-associated molecular patterns (PAMPs)-mediated autophagy of the endoplasmic reticulum [9]. In turn, STING could be also degraded by p62/SQSTM1-mediated autophagy [10]. A recent study showed that STING from the sea anemone Nematostella vectensis could trigger autophagy rather than the production of interferon when treated with cyclic GMP-AMP (cGAMP), indicating that autophagy triggered by STING is independent of IRF3 activation and may be an original function of the cGAS/STING signaling pathway [11]. Our recent study found that STING/IRF3 in cardiomyocytes could lead to sepsisinduced cardiac injury and dysfunction by activating NLRP3 inflammasomes [12]. However, the functions of STING as well as its regulatory mechanisms on autophagy in cardiac remodelling have not been well studied yet.

In this study, we examined whether STING overexpression affected cardiac hypertrophy, cardiac fibrosis, cardiac dysfunction and inflammation in the context of pressure overload. Meantime, we further elucidated the possible mechanisms of STING in cardiac remodelling.

\section{Materials and methods}

\section{Reagents and antibodies}

Rapamycin ( $\geq 99.93 \%$ purity, as determined by highperformance liquid chromatography), chloroquine (99.50\% purity), Angiotensin II (99.48\% purity) and TGF- $\beta$ were obtained from MCE (Shanghai, China). Primary antibodies against STING (1:1000), Beclin1 (1:1000), AMPK alpha 1+AMPK alpha 2 (1:1000), phosphorylated-AMPK alpha $1^{\text {T183 }}$ (p-AMPK alpha $1^{\text {T183 }}$ ) + phosphorylated-AMPK alpha $2^{\text {T172 }}$ (p-AMPK alpha $\left.2^{\text {T172}}\right)(1: 1000)$, mTOR (1:1000), phosphorylated-mTOR (p-mTOR $\left.{ }^{\mathrm{S} 2448}\right)$ (1:1000) were bought from Abcam (Cambridge, UK). Antibodies for Atg7 (1:1000), Atg12 (1:1000), LC3B (1:1000), LC3I//II (1:1000), GAPDH (1:1000), total ULK1 (1:1000), and phosphorylated-ULK1 $1^{\text {ser757 }}$ (p-ULK1 $1^{\text {ser757, }} 1$ 1:1000) were purchased from Cell Signaling Technology (Danvers, MA, USA). Anti-rabbit/mouse EnVisionTM+/HRP reagent for immunohistochemical staining was purchased from Gene Technology (Shanghai, China) and secondary antibody used for immunoblotting was obtained from LI-COR Biosciences.

\section{Animals and treatment}

The cardiomyocyte-specific overexpressed STING transgenic (TG) mice, constructed by integrating the STING target gene into the genome of wild type (WT) C57/B6 mice using microinjection, were purchased from the Model Animal Research Center of Nanjing University, Nanjing, China. Male C57/B6 mice were purchased from the Institute of Laboratory Animal Science, Chinese Academy of Medical Sciences (Beijing, China). The WT mice and TG mice used in this study were 8-10 weeks old with body weight $25.3 \pm 6$ g. All animal experimental procedures were approved by the Animal Experimentation Ethics Committee of Wuhan University (Protocol No. 00013274) and comply with the Guide for the Care and Use of Laboratory Animals by the US National Institutes of Health (NIH Publication No. 85-23, revised in 1996).

The mice were subjected to aortic banding (AB) surgery or sham operation as described previously [13].

The mice hearts and lungs were harvested and weighed after they were sacrificed using sodium pentobarbital. Then data involving the ratios of heart weight/ body weight $(\mathrm{HW} / \mathrm{BW}, \mathrm{mg} / \mathrm{g})$, lung weight/body weight (LW/BW, $\mathrm{mg} / \mathrm{g}$ ), and heart weight/tibia length (HW/ $\mathrm{TL}, \mathrm{mg} / \mathrm{mm}$ ) was collected.

To activate autophagy, Rapamycin $(2.0 \mathrm{mg} / \mathrm{kg})$ was used via intraperitoneal injection every other day for 4 weeks before $A B$ surgery. 


\section{Echocardiography}

On the basis of the previous study, transthoracic echocardiography was used to collect the mean echocardiographic parameters of 3 to 5 cardiac cycles, including heart rate (BPM), ejection fraction, ejection fraction, left ventricular end-diastolic diameter (LVEDD) and left ventricular end-systolic diameter (LVESD) [13].

\section{Histological analysis}

Simply put, the mouse heart was fixed with $4 \%$ formaldehyde overnight, then embedded in paraffin to make 5 - $\mu \mathrm{m}$ sections. Next, according to our previous study, hematoxylin eosin (H\&E) staining and Picrosirius Red (PSR) staining were carried out [14], aiming to observe the sectional area of cardiomyocytes and collagen volume in cardiac tissue.

Immunohistochemical staining was carried out to evaluate the content of inflammatory cells infiltration and LC3B. Endogenous peroxidase was inhibited by 3\% hydrogen peroxide and the nonspecific binding of the antibody were blocked with $10 \%$ goat serum. Then, the sections were incubated with anti-CD45 (1:100), antiCD68 (1:200), or anti LC3B (1:100) overnight at $4{ }^{\circ} \mathrm{C}$ as described previously [12].

\section{Cell culture and treatment}

Neonatal mice cardiomyocytes as well as cardiac fibroblasts were isolated and cultured based on previous description $[15,16]$. To overexpress STING, cardiomyocytes and cardiac fibroblasts were transfected with Ad-STING $(\mathrm{MOI}=10)$ or adenovirus harboring no overexpression sequence (Ad-Ctrl) for $6 \mathrm{~h}$. When the cells reached $75 \%$ confluence, cardiomyocytes were treated with AngII $(1 \mu \mathrm{M})$ while cardiac fibroblasts were treated with TGF- $\beta$ (10 ng/ml) for $24 \mathrm{~h}$. Experiments were performed at least 3 times in duplicate. To knock down the expression of STING, cardiomyocytes and cardiac fibroblasts were incubated with STING siRNA, as well as the scrambled siRNA (designed and synthesized by Sangon Biotech, Shanghai, China). Cardiomyocytes and cardiac fibroblasts in our study were transfected using Lipofectamine 3000 (Thermo Fisher Scientific, Waltham, MA, USA) based on the manufacturer's instructions. When the cells reached $75 \%$ confluence, Cardiomyocytes and cardiac fibroblasts were treated with Ang II or TGF- $\beta$ for $24 \mathrm{~h}$ in the presence/absence of STING. Chloroquine $(10 \mu \mathrm{M})$ was added $2 \mathrm{~h}$ before Ang II or TGF- $\beta$ stimulation [17].

\section{Western blot and real-time PCR}

Western blot was performed as our previously described $^{17}$. Extracting the total proteins of fresh ventricle tissues or iced cell lysates and quantified, approximately $50 \mu \mathrm{g}$ of total proteins were loaded on an SDS/PAGE gel. The resolved proteins were subsequently transferred onto polyvinylidene fluoride (PVDF) membranes. After that, membranes were put into 5\% non-fat milk with PBS/0.1\% Tween and blocked for $1 \mathrm{~h}$. Next, membranes containing target proteins were incubated with primary antibodies overnight at $4{ }^{\circ} \mathrm{C}$. The next day, after washing with $\mathrm{PBS} / 0.1 \%$ Tween, membranes were incubated with secondary antibodies conjugated to IRDye $800 \mathrm{CW}$ for $50 \mathrm{~min}$. Finally, images of the bands with target proteins were acquired and quantified by the Odyssey infrared imaging system (Odyssey, LI-COR, Lincoln, NE).

Real-time PCR was carried out as previously described [12]. The total RNA of the frozen ventricle tissues or iced cells was extracted by using TRIzol reagent (Invitrogen, 15596-026) and cDNA was synthesized using a Transcriptor First Strand cDNA Synthesis Kit (04896866001, Roche, USA). Quantitative real-time PCR was performed using Light Cycler 480 SYBR Green I Master Mix (Roche, 04707516001). The expression levels of target genes were uniformly normalized to GAPDH. The primers in realtime PCR are listed in Table 1.

\section{Immunofluorescent staining}

To further access cardiomyocyte hypertrophy and the transformation of cardiac fibroblast to myofibroblast, immunofluorescent staining for $\alpha$-actin and $\alpha$-SMA was performed according to our published article [15]. Briefly, the cardiomyocytes or cardiac fibroblasts were fixed using $4 \%$ paraformaldehyde and permeabilized using Triton X-100 (0.2\%). Cardiomyocyte hypertrophy was assessed by anti- $\alpha$-actinin staining while the transformation of cardiac fibroblast to myofibroblast was evaluated by anti- $\alpha$-SMA staining. DAPI dye was used to visualize cell nucleus. In addition, LC3B was also measured in the two sets to evaluate autophagy level.

\section{Statistical analysis}

All data are presented as mean \pm SD and analyzed with the software SPSS 23.3. Two-way analysis of variance (ANOVA) followed by Tukey's test for multiple comparisons while Student's unpaired t-test was applied to compare 2 groups. $P$ value $<0.05$ was regarded as statistically significant.

\section{Results \\ STING was upregulated in cardiac remodelling}

Firstly, the protein and mRNA level of STING was detected in mice heart challenged with pressure overload for different durations. The results showed the protein level of STING was significantly increased from 2 weeks 
Table 1 Primers in real-time PCR

\begin{tabular}{|c|c|c|c|}
\hline Gene & Species & Forward primer & Reverse primer \\
\hline $\mid L-1 \beta$ & Mouse & AATGAAGGAACGGAGGAGCC & CTCCAGCCAAGCTTCCTTGT \\
\hline IL-6 & Mouse & GTTGCCTTCTTGGGACTGATG & ATACTGGTCTGTTGTGGGTGGT \\
\hline MCP-1 & Mouse & TGGCTCAGCCAGATGCAGT & CCAGCCTACTCATTGGGATCA \\
\hline HMGB1 & Mouse & CCGGCAAGTTTGCACAAAGA & TTGGGAGGGCGGAGAATCAA \\
\hline GAPDH & Mouse & ACTCCACTCACGGCAAATTC & TCTCCATGGTGGTGAAGACA \\
\hline ANP & Mouse & ACCTGCTAGACCACCTGGAG- & CCTTGGCTGTTATCTTCGGTACCGG \\
\hline Collagen1 & Mouse & AGGCTTCAGTGGTTTGGATG & CACCAACAGCACCATCGTTA \\
\hline Collagen3 & Mouse & CCCAACCCAGAGATCCCATT & GAAGCACAGGAGCAGGTGTAGA \\
\hline Fibronectin & Mouse & CGGTGGCTGTCAGTCAGA & TCCCACTGCTGATTTATC \\
\hline CTGF & Mouse & TGTGTGATGAGCCCAAGGAC & AGTTGGCTCGCATCATAGTTG \\
\hline STING & Mouse & ATCTATGCTAGTCGTAGTTTA & CGTAGTGCTAGTGATTAGTC \\
\hline ANP & Rat & ATGGGCTCCTTCTCCATCAC & TCTTCGGTACCGGAAGCTG \\
\hline BNP & Rat & TTCCTTAATCTGTCGCCGCTGG & CAGCAGCTTCTGCATCGTGGAT \\
\hline Collagen 1 & Rat & GAGAGAGCATGACCGATGGATT & TGGACATTAGGCGCAGGAA \\
\hline Collagen3 & Rat & CAGACGGGAGTTTCTCCTCGGA & GACCAGGAGGACCAGCAACTCC \\
\hline GAPDH & Rat & GACATGCCGCCTGGAGAAAC & AGCCCAGGATGCCCTTTAGT \\
\hline
\end{tabular}
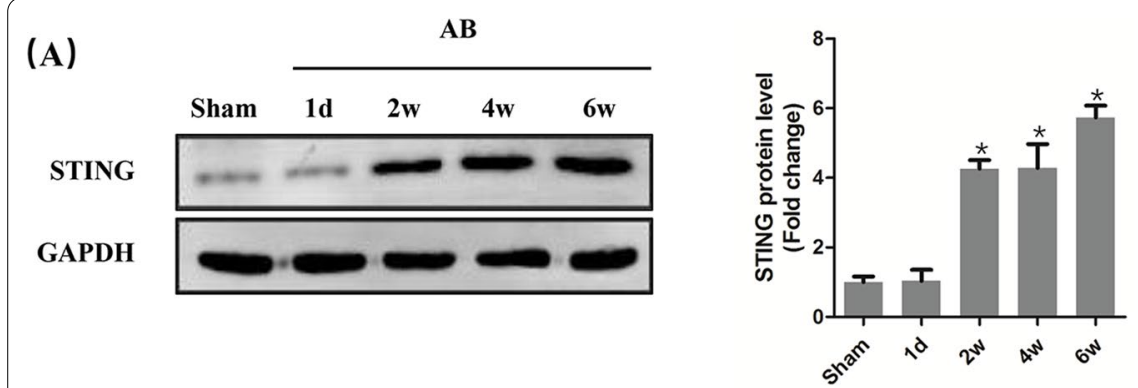

(B)

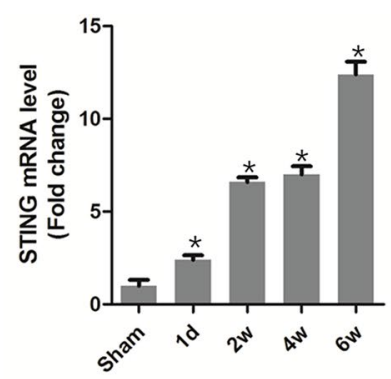

(C)

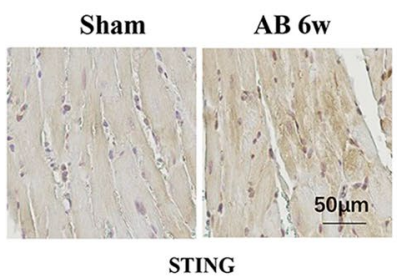

(D)

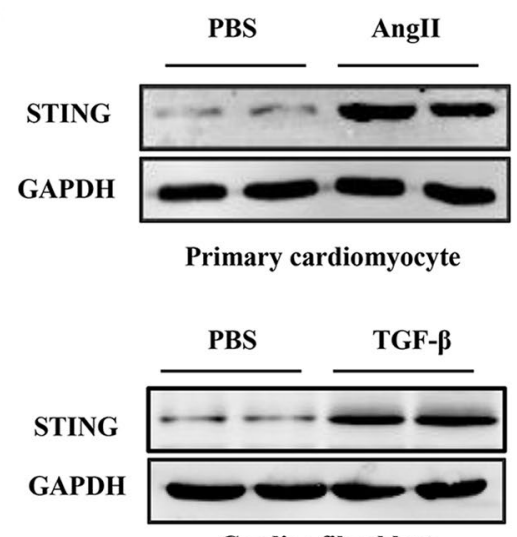

Cardiac fibroblast
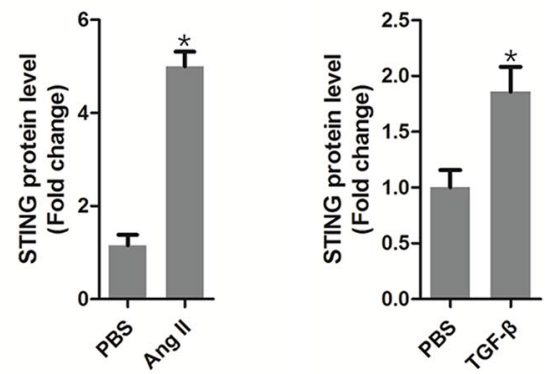

Fig. 1 STING was upregulated in the heart in AB-induced mice, Angll-stimulated primary cardiomyocytes and TGF- $\beta$-stimulated cardiac fibroblasts. A Representative western blot and analysis of STING in AB-induced hearts $(n=6)$. B The mRNA analysis of STING in AB-induced hearts $(n=6)$. C Immunohistochemistry of STING in AB-induced hearts $(n=6)$. D Representative western blot and analysis of STING in Angll-stimulated primary cardiomyocytes and TGF- $\beta$-stimulated cardiac fibroblasts $(n=6) .{ }^{*} P<0.05$ vs the Sham or PBS group

for at least 6 weeks after $A B$ surgery (Fig. 1A) while the mRNA level of STING started to increase from the first day after $A B$ surgery (Fig. 1B). Immumohistochemical staining further proved that STING was significantly upregulated at the sixth week after AB surgery (Fig. 1C). In vitro experiment further demonstrated that the 
protein expression of STING significantly increased in cardiomyocytes induced by Ang II or cardiac fibroblasts induced by TGF- $\beta$ (Fig. 1D).

\section{STING overexpression suppressed cardiac hypertrophy and fibrosis in vivo}

STING TG mice was used in this study and we used western blot to confirm that STING was overexpressed in TG mice (Fig. 2A). Subsequently, the mice were subjected to AB surgery for six weeks. As shown in Fig. 2B$\mathrm{D}$, the value of heart weight $(\mathrm{HW}) /$ body weight $(\mathrm{BW})$ and $\mathrm{HW} /$ tibia length (TL) significantly increased in $\mathrm{AB}+\mathrm{WT}$ group while STING overexpression could significantly inhibit them. It is worth noting that $A B$ and/ or STING overexpression did not affect the value of lung weight $(\mathrm{LW}) / \mathrm{BW}$ in mice. Furthermore, H\&E staining was used to assess the heart size as well as cross-sectional area of cardiomyocyte. As expected, STING overexpression significantly repressed the increase of heart size and cardiomyocyte size caused by pressure overload (Fig. 2E). Meanwhile, the marker of cardiac hypertrophy (ANP) could also significantly decrease in mice with $A B$ after STING was upregulated (Fig. 2F). PSR staining was used to evaluate the condition of fibrosis in the indicated groups. As shown in Fig. 2G, the percentage of perivascular fibrosis and interstitial fibrosis was significantly enhanced in WT mice while STING TG mice displayed obviously mitigation in both perivascular fibrosis and interstitial fibrosis after AB surgery for 6 weeks. Furthermore, marks of fibrosis involving fibronectin, ctgf, collagen1 and collagen3 were detected using real-time PCR. Compared with the mice in $\mathrm{AB}+\mathrm{WT}$ group, the levels of fibronectin, ctgf, collagen1 and collagen3 were significantly inhibited in $\mathrm{AB}+\mathrm{TG}$ group (Fig. $2 \mathrm{H}-\mathrm{K}$ ).

\section{STING overexpression mitigated cardiac inflammatory response}

Additionally, we investigated the cardiac inflammatory response in four groups. As shown in Fig. 3A, compared with WT mice challenged with $A B$ surgery, the percentage of CD45 positive cells (leukocytes) and CD68 positive cells (macrophages) significantly reduced in TG mice challenged with $A B$ surgery. Meanwhile, compared with $A B+W T$ group, the mRNA levels of proinflammatory cytokines including MCP-1, IL-6, IL-1 $\beta$ and HMGB1 were significantly inhibited in $\mathrm{AB}+\mathrm{TG}$ group (Fig. 3B-E).

\section{STING overexpression was involved in better cardiac function}

Next, we examined whether STING overexpression could affect cardiac function in mice subjected to $A B$ surgery. Pressure overload and/or STING overexpression had no effects on heart rate of mice (Fig. 4A). Mice displayed deteriorative cardiac function, reflected by reduced ejection fraction and fractional shortening in response to 6-week $A B$ surgery, which were significantly improved when STING was upregulated (Fig. 4B, C). Also, we detected the cardiac left ventricular end diameter at diastole and systole. As expected, the levels of left ventricular end-diastolic diameter (LVEDD) and left ventricular endsystolic diameter (LVESD) were significantly increased in STING TG mice after AB surgery for 6 weeks (Fig. 4D, E). The images of echocardiography also revealed that STING overexpression abolished the left ventricle enlargement induced by pressure overload (Fig. 4F).

\section{STING blocked cardiomyocyte hypertrophy and cardiac fibroblast activation in vitro}

Next, we confirmed the anti-remodelling effect of STING using adenovirus in vitro. The expression of STING was significantly enhanced in cardiomyocytes transfected with Ad-STING (Fig. 5A). STING overexpression significantly inhibited the mRNA levels of BNP as well ANP (Fig. 5B, C) and the cell cross-sectional area (Fig. 5D) of cardiomyocytes stimulated by Ang II. Similarly, the protein level of STING was significantly increased by AdSTING in cardiac fibroblasts (Fig. 5E). Fibrotic markers involving Collagen 1, Collagen 3 as well as $\alpha$-SMA were also suppressed in TGF- $\beta$-induced cardiac fibroblasts after STING was upregulated (Fig. 5F-H), indicating that STING not only inhibited collagen synthesis, but also repressed the transformation of cardiac fibroblasts to myofibroblasts.

\section{The cardioprotective effect of STING was mediated by autophagy inhibition}

Subsequently, we further explored the potential mechanism involved in the anti-remodelling effect of STING. Previous study has demonstrated that autophagy could be triggered by STING independent of IRF3 [18]. Hence, we next investigated whether STING overexpression affected myocardial autophagy activation of mice in response to pressure overload for 6 weeks. As shown in Fig. 6A, B, the protein levels of Beclin-1, Atg7, Atg12, as well as the ratio of LC3II/I were increased in mice challenged with $A B$ surgery, which were repressed after STING was upregulated. Rapamycin is one of the potent autophagy agonists through modulating mTOR [19]. The intraperitoneal injection of Rapamycin gave rise to the increase of Beclin-1(Fig. 6C). Additionally, STING TG mice challenged with pressure overload exhibited exacerbated cardiac function when autophagy was activated (Fig. 6D, E). Meanwhile, Rapamycin also abolished the anti-hypertrophy and anti-fibrosis effects of STING 


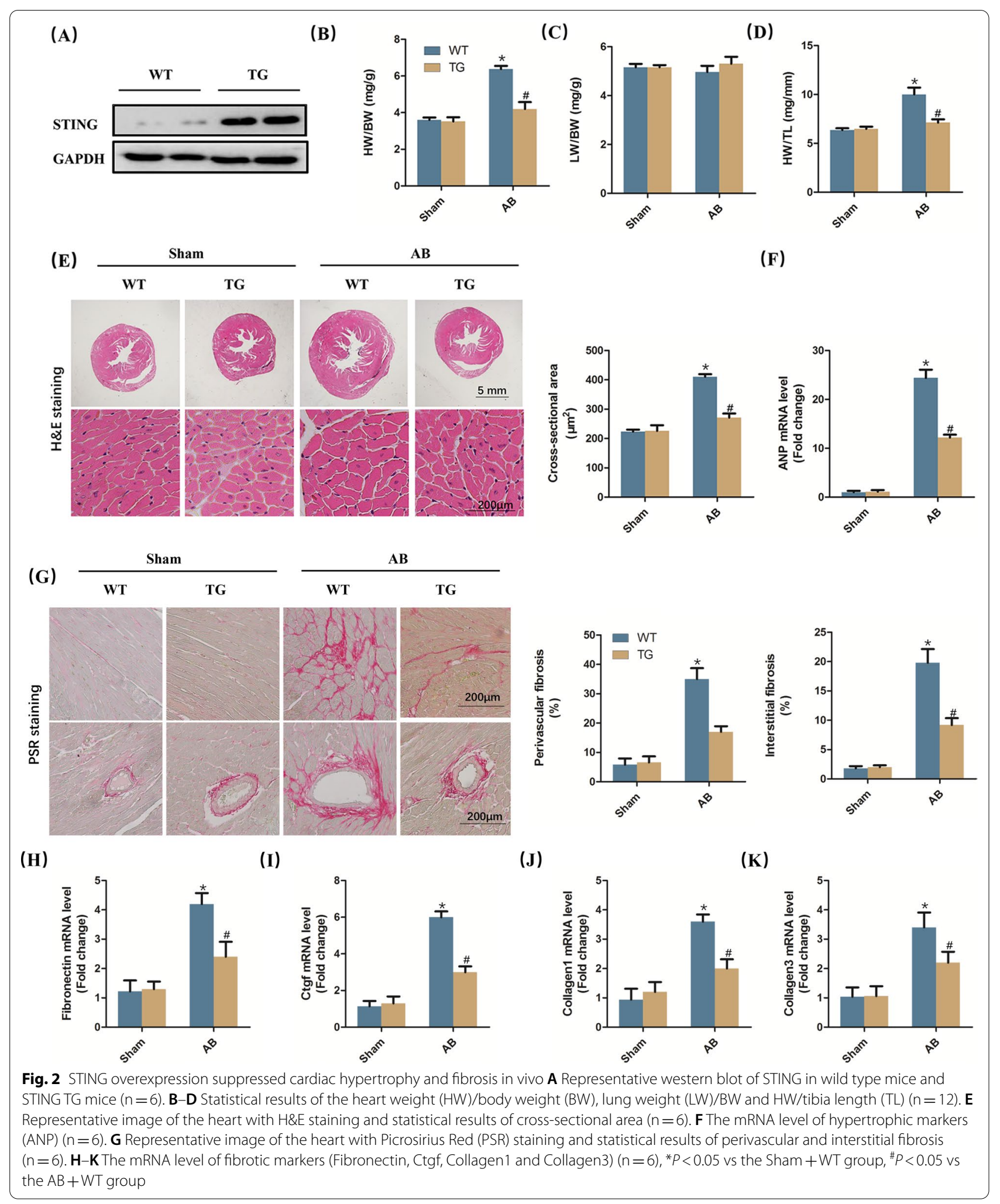



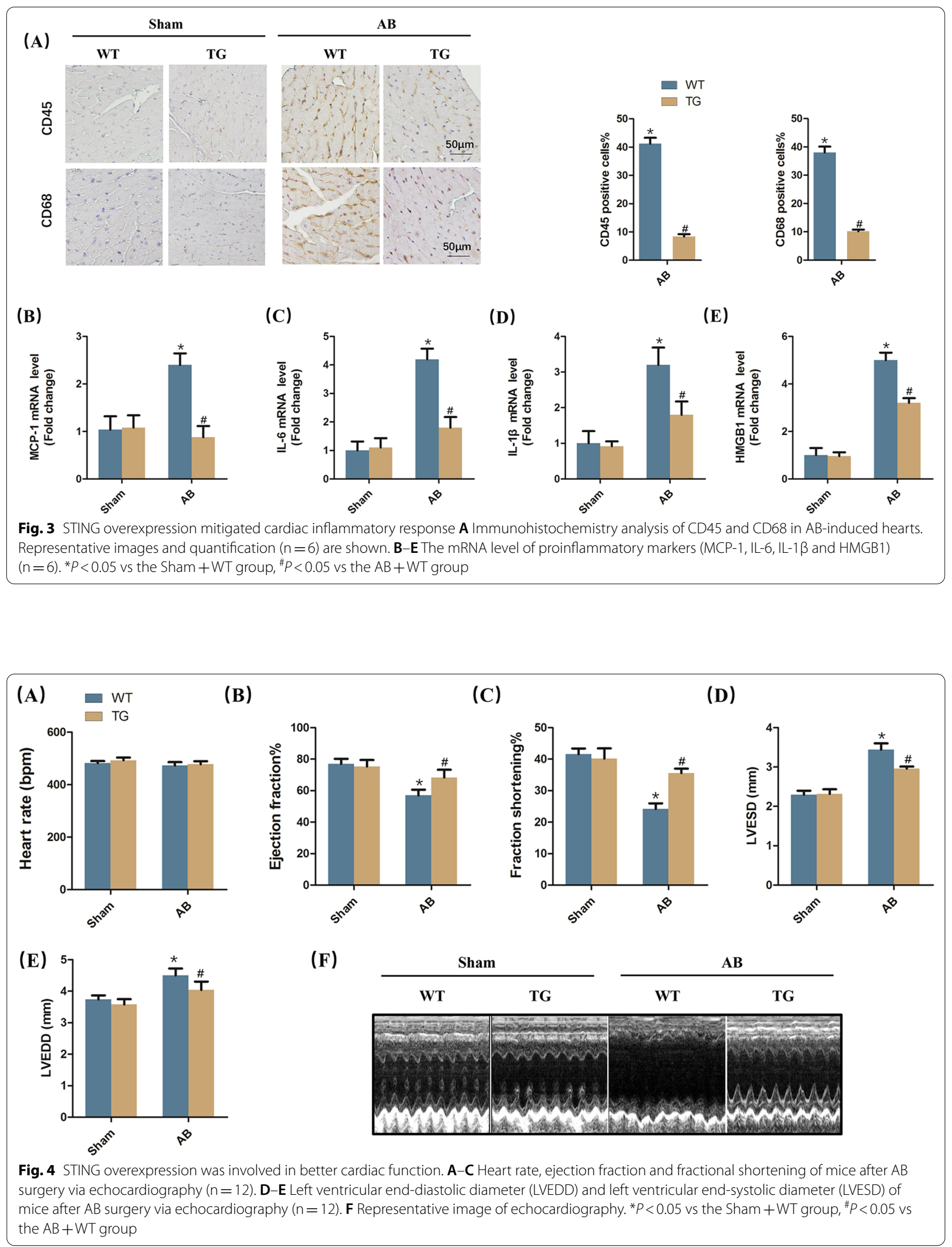
(A)

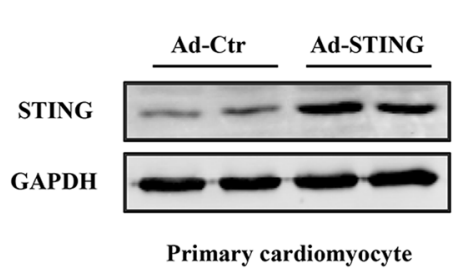

(D)

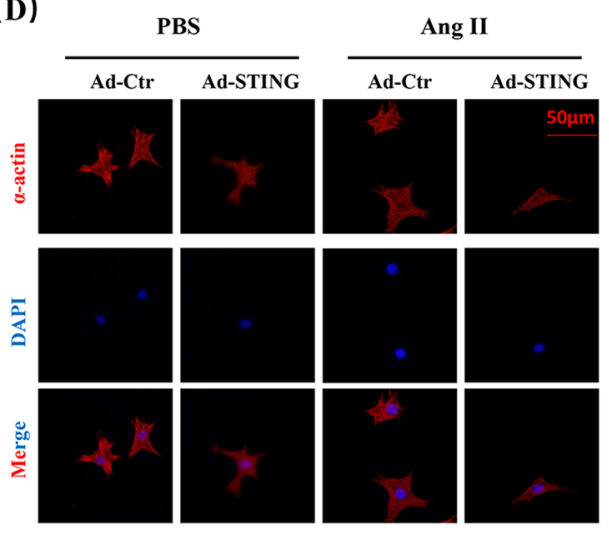

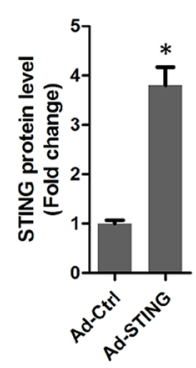
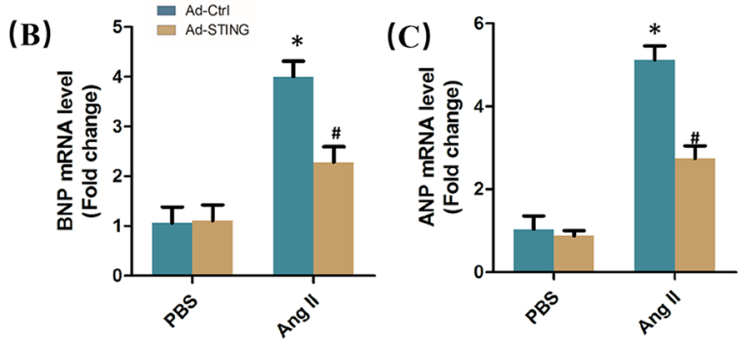

(E)

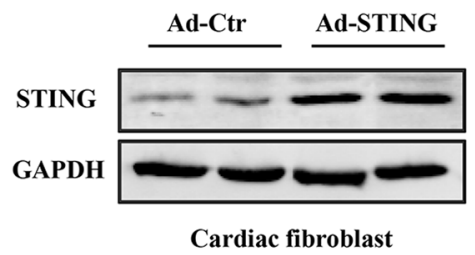

(H)

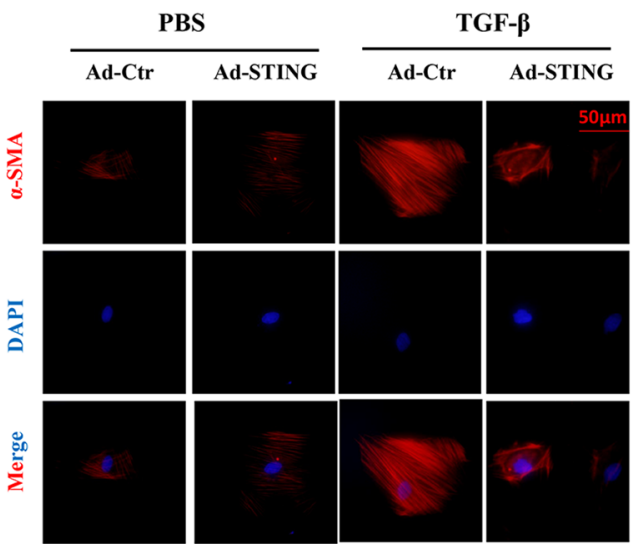

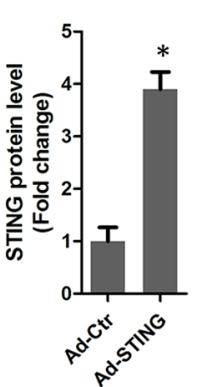

(F)

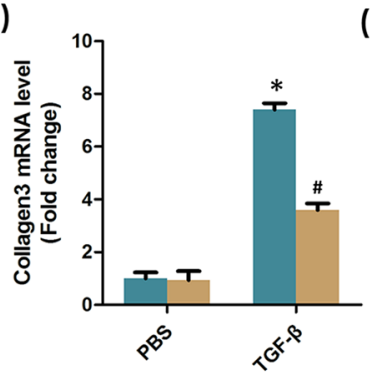

(G)

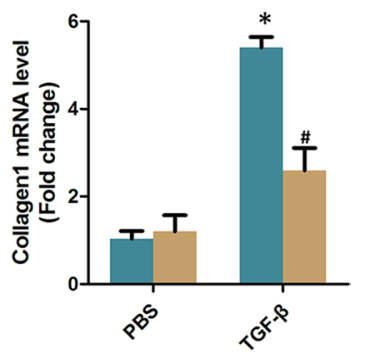

Fig. 5 STING blocked cardiomyocyte hypertrophy and cardiac fibroblast activation in vitro. A Representative western blot of STING in primary cardiomyocytes transfected with Ad-STING $(n=6) * P<0.05$ vs Ad-Ctrl. B, C The mRNA level of hypertrophic markers (BNP and ANP) $(n=6)$. D Immunofluorescence staining and analysis of $a$-actin in cardiomyocytes exposed to Angll and infected with Ad-STING $(n=6) * P<0.05$ vs PBS + Ad-Ctrl group, ${ }^{"} P<0.05$ vs Ang II + Ad-Ctrl group. E Representative western blot of STING in cardiac fibroblasts transfected with Ad-STING $(n=6) * P<0.05$ vs Ad-Ctrl. $\mathbf{F}$, G The mRNA level of fibrotic markers (Collagen 1 and Collagen3) $(n=6)$. $\mathbf{H}$ Immunofluorescence staining and analysis of a-SMA in cardiac fibroblasts exposed to TGF- $\beta$ and infected with Ad-STING $(n=6)$, ${ }^{*} P<0.05$ vs PBS + Ad-Ctrl group, ${ }^{\#} P<0.05$ vs Ang $\|+$ Ad-Ctrl group 


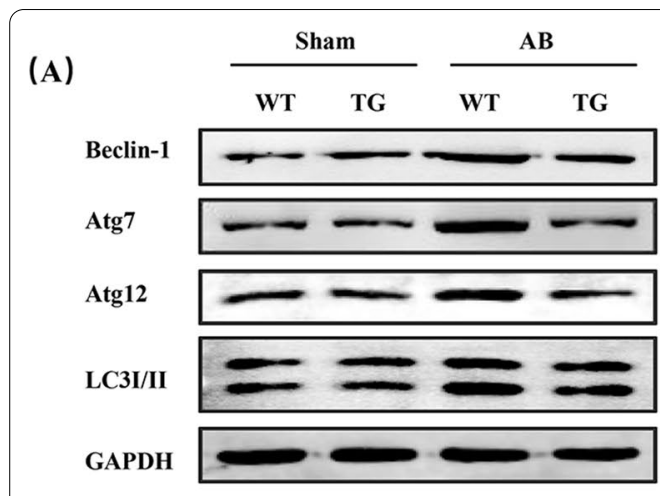

(C)

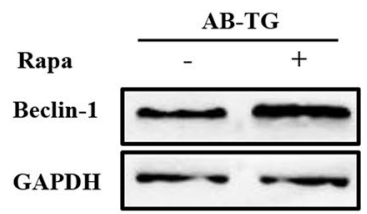

(G)
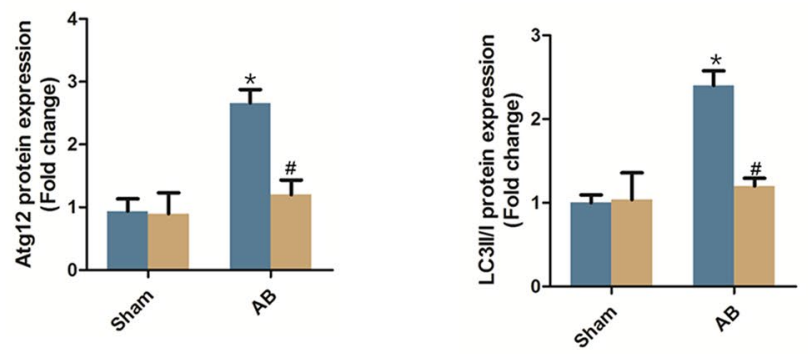

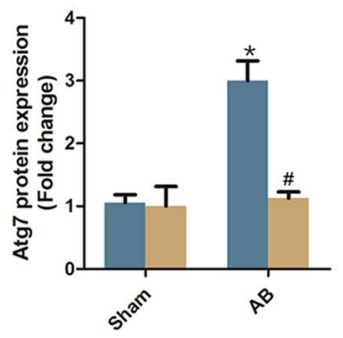

(B)

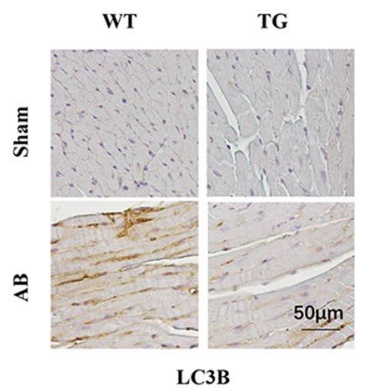

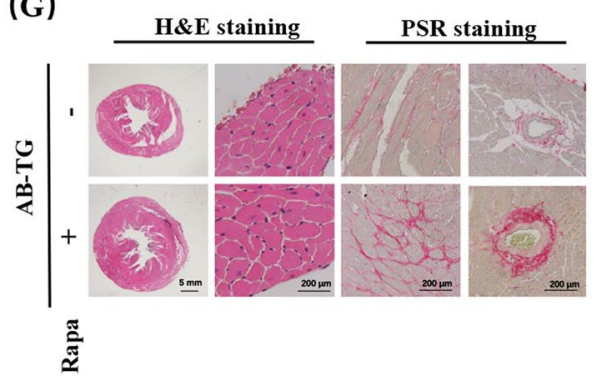

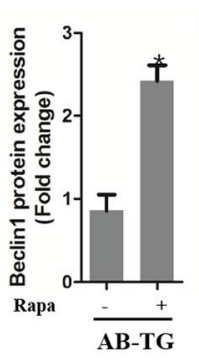

(D)
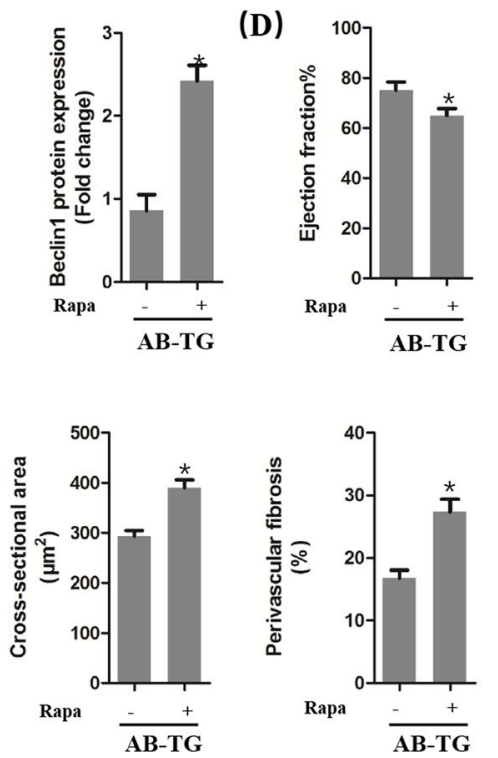

(E)

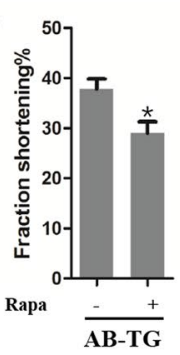

(F)

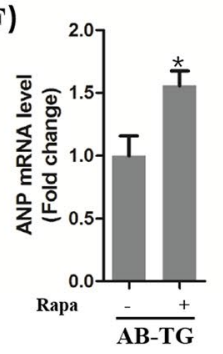

(H)
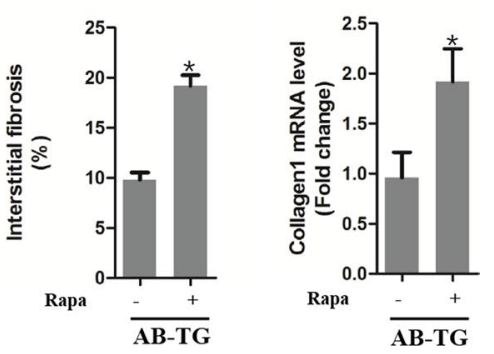

Fig. 6 The cardioprotective effect of STING was mediated by autophagy inhibition. A Representative western blot and analysis of Beclin-1, Atg7, Atg1 2 and $L C 3 I / I I(n=6)$. ${ }^{*} P<0.05$ vs the Sham + WT group, ${ }^{\#} P<0.05$ vs the $A B+W T$ group. $B$ Immunohistochemistry analysis of $L C 3 B$ in indicated groups. C Representative western blot and analysis of Beclin-1 in STING TG mice with AB and rapamycin $(n=6)$. D, E Ejection fraction and fractional shortening in STING TG mice with AB and rapamycin via echocardiography $(n=12)$. F The mRNA level of hypertrophic markers (ANP) $(n=6)$. G Representative image of the heart with H\&E staining and Picrosirius Red (PSR) staining and statistical results $(n=6)$. $\mathbf{H}$ The mRNA level of fibrotic markers (Collagen 1$)(n=6),{ }^{*} P<0.05$ vs AB-TG group

in vivo (Fig. 6F-H). While in vitro, STING overexpression significantly inhibited the LC3B of cardiomyocytes stimulated by Ang II (Additional file 1:
Figure 1A-B). Similarly, LC3B were also suppressed in TGF- $\beta$-induced cardiac fibroblasts after STING was upregulated (Additional file 1: Figure 1C-D). These 
further proved that STING protected against cardiac remodelling and dysfunction in an autophagy inhibition manner.

\section{STING may inhibit autophagy through phosphorylating ULK1 in heart}

ULK1 activation is the first step to initiate autophagy [20]. Next, we explored whether STING inhibited autophagy in heart was involved in the phosphorylation of ULK1. Western blot showed that myocardial phosphorylation level of ULK1 in mice subjected to $A B$ surgery was significantly inhibited. However, STING significantly increased the phosphorylation level of ULK1 (Fig. 7), suggesting that ULK1 was implicated with the cardioprotective effects of STING. Considering AMPK/mTOR is the upstream signal of ULK1 [21], we further investigated whether STING promotes the phosphorylation of ULK1 by affecting the AMPK/ mTOR axis. We successfully knocked down STING in cardiomyocytes (Additional file 1: Figure 2A-B). The results showed that chloroquine, a lysosomal inhibitor, inhibited the phosphorylation of AMPK, promoted the phosphorylation of mTOR and ULK1 in cardiomyocytes with Ang II stimulation. After STING was knocked down, the phosphorylation of ULK1 decreased significantly, but the phosphorylation of AMPK and mTOR were not affected (Additional file 1: Figure $2 \mathrm{C}-\mathrm{F}$ ). We further successfully knocked down STING in cardiac fibroblasts (Additional file 1: Fig. 2G-H). The results presented a similar conclusion with cardiomyocytes (Additional file 1: Fig. 2I-L). These indicating that STING phosphorylates ULK1 in an AMPK/mTOR-independent manner.

\section{Discussion}

Our study disclosed the cardioprotective potential of STING in pressure overload-induced cardiac remodelling, using a realistic model of human heart failure. In detail, STING could participate in cardioprotection from the following aspects: (1) reduction of cardiac fibrosis and cardiac hypertrophy; (2) suppression of proinfammatory cytokines production; (3) improvement of cardiac function; (4) inhibition of autophagy; (5) phosphorylation of ULK1.

As a conserved, homeostatic process for the degradation and recycle of intracellular damaged organelles and macromolecules, autophagy has been proved to exert essential roles in improve cardiac function with cardiac remodeling [22]. In fact, the role of autophagy in cardiac hyperthrophy and heart failure is still controversial. Cardiac-specific Atg5 deficiency mice exhibited worse cardiac function and more serious cardiac hypertrophy after pressure overload for 1 week compared with wild type mice [23]. Meanwhile, Atg5 null mice displayed significantly less suppression of left ventricle hypertrophy than the control mice after 7 days, unloading [24]. One clinical research also demonstrated that myofilament alterations and autophagic vacuoles was associated with better prognosis in patients with dilated cardiomyopathy, indicating that autophagy activation may serve as an adaptive response [25]. On the contrary, Beclin-1 overexpression or some pharmacological agonists aggravated cardiac hypertrophy by activating autophagy [6,26], which implied that excessive autophagy may give cell death and act as an undesirable role in maintain cardiac function. Accumulating evidence unveiled that both excessive and insufficient autophagy could trigger cardiac hypertrophy for the reason that the dynamic balance between cardiac

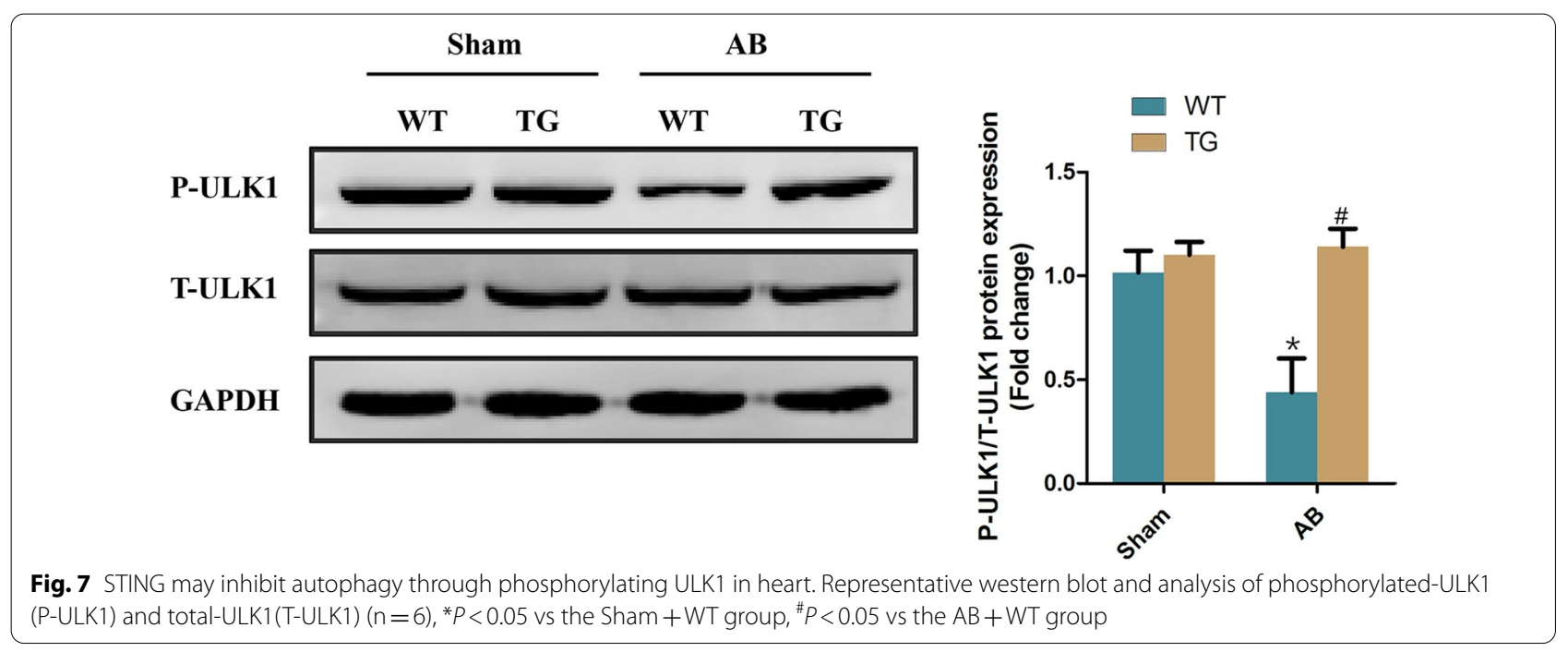


protein degradation and synthesis determines myocardial mass [6]. As for cardiac fibrosis, the maladaptive effect of autophagy is relatively defined. Studies have shown that TGF- $\beta$ could promote autophagy in human tubular epithelial kidney cells and mouse fibroblasts $[27,28]$. In human atrial myofibroblasts, TGF- $\beta$ may simultaneously trigger autophagy and fibrosis in human atrial myofibroblasts and pharmacological inhibition of autophagy caused the decrease in TGF- $\beta$-induced cardiac fibrosis [29]. In our study, autophagy was activated in mice subjected to pressure overload, which was accompanied with deteriorated hypertrophic growth, worse cardiac function, increased fibrosis as well as inflammatory response. However, autophagy inhibition significantly repressed pathological alteration of heart, hinting that autophagy activation may play a negative role in the pathogenesis of chronic heart failure.

STING protein is located on the endoplasmic reticulum, which was firstly discovered in 2008 . The original function of STING is to promote type I IFN production upon the stimulation cyclic dinucleotides. In recent years, STING has been recognized as a critical and promising target for various cardiovascular diseases. For instance, STING-IFRF3 axis is critically associated in obesity-induced endothelial injury and inflammation, the inhibition of which could partially protected against adipose tissue inflammation, insulin resistance, obesity, as well as glucose intolerance [30]. In the model of myocardial infarction, suppression of IRF3dependent signaling leaded to reduced levels of cardiac inflammatory cytokines and decreased inflammatory cell infiltration, apart from relieving left ventricular dilation and improving cardiac function [31]. Our lab previously reported that STING-IRF3 axis could be initiated by lipopolysaccharide, thus trigger cardiomyocytes apoptosis and pyroptosis by activating NLRP3 inflammasome [12]. In the present study, STING overexpression significantly improved cardiac function, and alleviated $\mathrm{AB}$-induced cardiac hypertrophy, fibrosis as well as inflammation. The abnormal protein synthesis within the endoplasmic reticulum could lead to the accumulation of unfolded proteins, thereby giving rise to endoplasmic reticulum stress as well as the unfolded protein response. Endoplasmic reticulum stress has been identified one of the main mechanisms contributing to cardiac remodeling [32]. In hepatocytes, endoplasmic reticulum stress could activate IRF3 via STING, eventually causing hepatocyte apoptosis [8]. Clinical study has disclosed that endoplasmic reticulum stress is activated during cardiac remodelling, which exerts a crucial role in maintaining cell homeostasis [8]. Endoplasmic reticulum stress is also linked to the autophagic response and the endoplasmic reticulum stress-autophagy pathway is involved in pathological cardiac hypertrophy [33]. Based on these studies, we speculate that STING may serve as a bridge between endoplasmic reticulum stress and autophagy.

Apoptotic signaling-dependent disruption of ULK1, a pro-autophagic protein negatively regulating STING, could stimulate STING-dependent IRF3 activation by phosphorylating STING at serine 366 site [34, 35], indicating that STING could directly interact with ULK1. On the other hand, mTOR can phosphorylate ULK1 at its serine 757 site and inhibit its kinase activity, thus blocking autophagy [36]. Considering that AMPK/mTOR/ ULK1 axis is deeply involved in autophagy process [21], we further investigated whether STING promotes the phosphorylation of ULK1 at its serine 757 site by affecting the AMPK / mTOR axis. After STING was knocked down in cardiomyocytes or cardiac fibroblasts, the phosphorylation of ULK1 decreased significantly, but the phosphorylation of AMPK and mTOR were not affected in Ang II or TGF- $\beta$ + chloroquine group. These indicating that STING phosphorylates ULK1 in an AMPK / mTOR-independent manner.

It is worth noting that a relatively recent publication by Zhang et al. [37] identifies STING as a critical mediator of cardiac remodelling and ER stress, where its suppression was found to be associated with improved outcomes. Some reasons may explain the controversy. Firstly, in our study, we proved the protective effects of STING overexpression on cardiac function in cardiomyocyte-specific overexpressed STING mice, which indicated from the side that low expression or deletion of STING in cardiomyocytes would have adverse effects on cardiac function. Wang et al. also disclosed that STING suppression were harmful to cardiac function [38]. While in Zhang et al's study, STING conventional knockout mice were used in TAC model. We think that the loss of STING in cardiomyocytes is indeed detrimental to cardiac function, but the loss of STING in other tissues and cells may have a series of direct or indirect protective effects on the heart, and such protective effects are greater than the adverse factors. Therefore, their research showed that STING suppression had protective effects on cardiac function, which is the opposite of what we found. Maybe further experiments are needed to explain this phenomenon. More importantly, STING as an important element to activate innate immunity, plays an important role in immune cells, especially in macrophages $[39,40]$, so it is actually difficult to demonstrate the value of STING in cardiomyocytes in conventional knockout mice. Finally, the mice were subjected to $A B$ surgery for six weeks in our study, but only four weeks for their mice. Different modeling time may also cause inconsistencies in experimental results. 


\section{Conclusions}

In summary, we demonstrate that STING, an endoplasmic reticulum adaptor, may ameliorate the development of AB-induced cardiac fibrosis and hypertrophy by repressing autophagy. STING can increase the phosphorylation of ULK1, thus decreasing inflammation and autophagy in heart.

\begin{abstract}
Abbreviations
STING: Stimulator of interferon genes; AB: Aortic binding; NF-kB: Nuclear factor (NF)-KB; IRF3: Interferons (IFN) regulator 3; PAMPs: Pathogen-associated molecular patterns; CGAMP: Cyclic GMP-AMP; p-ULK1ser757: PhosphorylatedULK1ser757; WT: Wild type; TG: Transgenic; HW/BW: Heart weight/body weight; LW/BW: Lung weight/body weight; HW/TL: Heart weight/tibia length; LVEDD: Left ventricular end-diastolic diameter; LVESD: Left ventricular endsystolic diameter; H\&E: Hematoxylin eosin; PSR: Picrosirius Red.
\end{abstract}

\section{Supplementary Information}

The online version contains supplementary material available at https://doi. org/10.1186/s12964-021-00793-0.

Additional file 1. STING overexpression inhibited LC3B expression in vitro and STING phosphorylates ULK1 in an AMPK/mTOR-independent manner.

\section{Acknowledgements}

Not applicable.

\section{Authors' contributions}

QG and WJ designed the study that led to this article. RX and NL contributed to the experiments related to animals and cells, experimental detection, manuscript preparation and revision. LC contributed to revise the manuscript all the time. WW and BW contributed toward data analysis and drafting the manuscript. All authors read and approved the final manuscript.

\section{Funding}

This study was supported by grant from the National Natural Science Foundation of China (Grant No. 81770095).

\section{Availability of data and materials}

The datasets used and/or analysed during the current study are available from the corresponding author on reasonable request.

\section{Declarations}

\section{Ethics approval and consent to participate}

All animal experimental procedures were approved by the Animal Experimentation Ethics Committee of Wuhan University (Protocol No. 00013274) and comply with the Guide for the Care and Use of Laboratory Animals by the US National Institutes of Health (NIH Publication No. 85-23, revised in 1996).

\section{Consent for publication}

Not applicable.

\section{Competing interests}

The authors declare no conflicts of interest.

\section{Author details}

${ }^{1}$ Department of Thoracic Surgery, Renmin Hospital of Wuhan University, Wuhan, China. ${ }^{2}$ Department of Orthopedics, Renmin Hospital of Wuhan University, Wuhan, China.

Received: 29 April 2021 Accepted: 9 October 2021

Published online: 08 November 2021
References

1. Haque Z, Wang D. How cardiomyocytes sense pathophysiological stresses for cardiac remodeling. Cell Mol Life Sci: CMLS. 2017;74:983-1000.

2. Liu C, Tang W. Epigenetics in cardiac hypertrophy and heart failure. JACC Basic Transl Sci. 2019:4:976-93.

3. Wu Q, Xiao Y, Yuan Y, Ma Z, Liao H, Liu C, Zhu J, Yang Z, Deng W, Tang Q. Mechanisms contributing to cardiac remodelling. Clin Sci (London, England: 1979). 2017;131:2319-45.

4. Lee $\mathrm{H}, \mathrm{Oh} \mathrm{SH}, \mathrm{Cho} \mathrm{H}, \mathrm{Cho} \mathrm{HJ}$, Kang HY. Prevalence and socio-economic burden of heart failure in an aging society of South Korea. BMC Cardiovasc Disord. 2016;16:215.

5. Schirone L, Forte M, Palmerio S, Yee D, Nocella C, Angelini F, Pagano F, Schiavon S, Bordin A, Carrizzo A, et al. A review of the molecular mechanisms underlying the development and progression of cardiac remodeling. Oxid Med Cell Longev. 2017;2017:3920195.

6. Nishida K, Otsu K. Autophagy during cardiac remodeling. J Mol Cell Cardiol. 2016;95:11-8

7. Li L, Xu J, He L, Peng L, Zhong Q, Chen L, Jiang Z. The role of autophagy in cardiac hypertrophy. Acta Biochim Biophys Sin. 2016;48:491-500.

8. Petrasek J, Iracheta-Vellve A, Csak T, Satishchandran A, Kodys K, Kurt-Jones E, Fitzgerald K, Szabo G. STING-IRF3 pathway links endoplasmic reticulum stress with hepatocyte apoptosis in early alcoholic liver disease. Proc Natl Acad Sci USA. 2013;110:16544-9.

9. Moretti J, Roy S, Bozec D, Martinez J, Chapman J, Ueberheide B, Lamming D, Chen Z, Horng T, Yeretssian G, et al. STING senses microbial viability to orchestrate stress-mediated autophagy of the endoplasmic reticulum. Cell. 2017;171:809-823.e813.

10. Prabakaran T, Bodda C, Krapp C, Zhang B, Christensen M, Sun C, Reinert $L$, Cai $Y$, Jensen S, Skouboe M, et al. Attenuation of CGAS-STING signaling is mediated by a p62/SQSTM1-dependent autophagy pathway activated by TBK1. EMBO J. 2018;37:e97858.

11. Gui X, Yang H, Li T, Tan X, Shi P, Li M, Du F, Chen Z. Autophagy induction via STING trafficking is a primordial function of the CGAS pathway. Nature. 2019;567:262-6.

12. Li N, Zhou H, Wu H, Wu Q, Duan M, Deng W, Tang Q. STING-IRF3 contributes to lipopolysaccharide-induced cardiac dysfunction, inflammation, apoptosis and pyroptosis by activating NLRP3. Redox Biol. 2019:24:101215.

13. Wu Q, Xiao Y, Liu C, Duan M, Cai Z, Xie S, Yuan Y, Wu H, Deng W, Tang Q. The protective effect of high mobility group protein HMGA2 in pressure overload-induced cardiac remodeling. J Mol Cell Cardiol. 2019;128:160-78

14. Li N, Zhou H, Ma Z, Zhu J, Liu C, Song P, Kong C, Wu H, Deng W, Tang Q. in vivoGeniposide alleviates isoproterenol-induced cardiac fibrosis partially via SIRT1 activation and. Front Pharmacol. 2018;9:854.

15. Ma Z, Yuan Y, Zhang X, Xu S, Wang S, Tang Q. Piperine attenuates pathological cardiac fibrosis via PPAR- $\gamma /$ AKT pathways. EBioMedicine. 2017;18:179-87.

16. Wu Q, Yuan Y, Jiang X, Xiao Y, Yang Z, Ma Z, Liao H, Liu Y, Chang W, Bian Z, Tang Q. OX40 regulates pressure overload-induced cardiac hypertrophy and remodelling via CD4+ T-cells. Clin Sci (London, England: 1979). 2016;130:2061-71.

17. Rahman MA, Cho Y, Nam G, Rhim H. Antioxidant compound, oxyresveratrol, inhibits APP production through the AMPK/ULK1/mTOR-mediated autophagy pathway in mouse cortical astrocytes. Antioxidants (Basel, Switzerland). 2021;10:408.

18. Caccamo A, Majumder S, Richardson A, Strong R, Oddo S. Molecular interplay between mammalian target of rapamycin (mTOR), amyloid-beta, and Tau: effects on cognitive impairments. J Biol Chem. 2010;285:13107-20.

19. Torii S, Yamaguchi H, Nakanishi A, Arakawa S, Honda S, Moriwaki K, Nakano H, Shimizu S. Identification of a phosphorylation site on Ulk1 required for genotoxic stress-induced alternative autophagy. Nat Commun. 2020;11:1754.

20. Kroemer G, Mariño G, Levine B. Autophagy and the integrated stress response. Mol Cell. 2010;40:280-93.

21. Luo Q, Song Y, Kang J, Wu Y, Wu F, Li Y, Dong Q, Wang J, Song C, Guo H. mtROS-mediated Akt/AMPK/mTOR pathway was involved in Copperinduced autophagy and it attenuates Copper-induced apoptosis in RAW264.7 mouse monocytes. Redox biology. 2021;41:101912.

22. Nakai A, Yamaguchi O, Takeda T, Higuchi Y, Hikoso S, Taniike M, Omiya S, Mizote I, Matsumura Y, Asahi M, et al. The role of autophagy in 
cardiomyocytes in the basal state and in response to hemodynamic stress. Nat Med. 2007;13:619-24.

23. Oyabu J, Yamaguchi O, Hikoso S, Takeda T, Oka T, Murakawa T, Yasui H, Ueda H, Nakayama H, Taneike M, et al. Autophagy-mediated degradation is necessary for regression of cardiac hypertrophy during ventricular unloading. Biochem Biophys Res Commun. 2013;441:787-92.

24. Saito T, Asai K, Sato S, Hayashi M, Adachi A, Sasaki Y, Takano H, Mizuno K, Shimizu W. Autophagic vacuoles in cardiomyocytes of dilated cardiomyopathy with initially decompensated heart failure predict improved prognosis. Autophagy. 2016;12:579-87.

25. Zhu H, Tannous P, Johnstone J, Kong Y, Shelton J, Richardson J, Le V, Levine B, Rothermel B, Hill J. Cardiac autophagy is a maladaptive response to hemodynamic stress. J Clin Investig. 2007;117:1782-93.

26. Su F, Shi M, Zhang J, Zheng Q, Zhang D, Zhang W, Wang H, Li X. Simvastatin protects heart from pressure overload injury by inhibiting excessive autophagy. Int J Med Sci. 2018;15:1508-16.

27. Xu Y, Yang S, Huang J, Ruan S, Zheng Z, Lin J. Tgf- $\beta 1$ induces autophagy and promotes apoptosis in renal tubular epithelial cells. Int J Mol Med. 2012;29:781-90

28. Kim S, Na H, Ding Y, Wang Z, Lee S, Choi M. Autophagy promotes intracellular degradation of type I collagen induced by transforming growth factor (TGF)-B1. J Biol Chem. 2012;287:11677-88.

29. Ghavami S, Cunnington R, Gupta S, Yeganeh B, Filomeno K, Freed D, Chen $\mathrm{S}$, Klonisch T, Halayko A, Ambrose $\mathrm{E}$, et al. Autophagy is a regulator of TGF- $\beta 1$-induced fibrogenesis in primary human atrial myofibroblasts. Cell Death Dis. 2015;6:e1696.

30. Mao Y, Luo W, Zhang L, Wu W, Yuan L, Xu H, Song J, Fujiwara K, Abe J, LeMaire S, et al. STING-IRF3 triggers endothelial inflammation in response to free fatty acid-induced mitochondrial damage in diet-induced obesity. Arterioscler Thromb Vasc Biol. 2017;37:920-9.

31. King K, Aguirre A, Ye Y, Sun Y, Roh J, Ng R, Kohler R, Arlauckas S, I wamoto Y, Savol A, et al. IRF3 and type I interferons fuel a fatal response to myocardial infarction. Nat Med. 2017:23:1481-7.

32. Dickhout J, Carlisle R, Austin R. Interrelationship between cardiac hypertrophy, heart failure, and chronic kidney disease: endoplasmic reticulum stress as a mediator of pathogenesis. Circ Res. 2011;108:629-42.
33. Guan H, Shangguan H, Shang Z, Yang L, Meng X, Qiao S. Endoplasmic reticulum stress caused by left ventricular hypertrophy in rats: effects of telmisartan. Am J Med Sci. 2011;342:318-23.

34. Xie F, Wu D, Huang S, Cao J, Li H, He L, Liu M, Li L, Chen L. The endoplasmic reticulum stress-autophagy pathway is involved in apelin13-induced cardiomyocyte hypertrophy in vitro. Acta Pharmacol Sin. 2017;38:1589-600.

35. Kemp M, Lindsey-Boltz L, Sancar A. UV light potentiates STING (Stimulator of Interferon Genes)-dependent innate immune signaling through deregulation of ULK1 (Unc51-like Kinase 1). J Biol Chem. 2015;290:12184-94.

36. Konno H, Konno K, Barber G. Cyclic dinucleotides trigger ULK1 (ATG1) phosphorylation of STING to prevent sustained innate immune signaling. Cell. 2013;155:688-98.

37. Zhang Y, Chen W, Wang Y. STING is an essential regulator of heart inflammation and fibrosis in mice with pathological cardiac hypertrophy via endoplasmic reticulum (ER) stress. Biomed Pharmacother. 2020;125:110022.

38. Wang S, Wang L, Qin X, Turdi S, Sun D, Culver B, Reiter R, Wang X, Zhou $H$, Ren J. ALDH2 contributes to melatonin-induced protection against APP/PS1 mutation-prompted cardiac anomalies through cGAS-STINGTBK1-mediated regulation of mitophagy. Signal Transduct Target Ther. 2020;5:119.

39. Motwani M, Pesiridis S, Fitzgerald K. DNA sensing by the CGAS-STING pathway in health and disease. Nat Rev Genet. 2019;20:657-74.

40. Feng $X$, Liu D, Li Z, Bian J. Bioactive modulators targeting STING adaptor in CGAS-STING pathway. Drug Discov Today. 2020;25:230-7.

\section{Publisher's Note}

Springer Nature remains neutral with regard to jurisdictional claims in published maps and institutional affiliations.
Ready to submit your research? Choose BMC and benefit from:

- fast, convenient online submission

- thorough peer review by experienced researchers in your field

- rapid publication on acceptance

- support for research data, including large and complex data types

- gold Open Access which fosters wider collaboration and increased citations

- maximum visibility for your research: over $100 \mathrm{M}$ website views per year

At BMC, research is always in progress.

Learn more biomedcentral.com/submissions 